\section{Condições socioeconômicas e padrões alimentares de crianças de 4 a 11 anos: estudo SCAALA - Salvador/ Bahia}

\section{The socio-economic conditions and patterns of food intake in children aged between 4 and 11 years: the SCAALA study - Salvador/ Bahia}

Silvana D'Innocenzo 1

Dirce M. L. Marchioni 2

Matildes S. Prado 3

Sheila M. A. Matos 4

Sheila R. S. Pereira 5

Antoniel P. Barros 6

Lílian R. Sampaio 7

Ana M. O. Assis 8

Laura C. Rodrigues 9

Maurício L. Barreto 10

1,3-6,10 Instituto de Saúde Coletiva. Universidade Federal da Bahia. Rua Basílio da Gama, s.n. Campus Universitário Canela. Salvador, BA, Brasil. CEP: 40.110-040

E-mail: silvanadinnocenzo@gmail.com

2 Departamento de Nutrição. Faculdade de Saúde Pública. Universidade de São Paulo. São Paulo, SP, Brasil.

7,8 Escola de Nutrição. Universidade Federal da Bahia. Salvador, BA, Brasil.

9 Department of Epidemiology and Population Health. London School of Hygiene and Tropical Medicine. University of London. London, UK.

\section{Resumo}

Objetivos: identificar os padrões alimentares de crianças e sua associação com o nível socioeconômico das familias.

Métodos: estudo transversal com 1260 crianças de 4 a 11 anos, residentes em Salvador-Bahia que incluiu aplicação de um Questionário de Frequência Alimentar semi-quantitativo. Os padrões alimentares foram identificados, empregando-se análise fatorial por componentes principais. O nível socioeconômico foi avaliado por meio de um indicador socioeconômico composto. Regressão logística multivariada foi empregada.

Resultados: identificaram-se quatro padrões que explicaram $45,9 \%$ da variabilidade dos dados de frequência alimentar. Crianças que pertencem ao nível socioeconômico mais alto têm 1,60 vezes mais chance $(p<0,001)$ de apresentarem maior frequência de consumo de alimentos do padrão 1 (frutas, verduras, leguminosas, cereais e pescados) e 3,09 vezes mais chance $(p<0,001)$ de apresentarem maior frequência de consumo dos alimentos do padrão 2 (leite/ derivados, catchup/ maionese) mostarda e frango), quando se compara com aquele de crianças de nivel socioeconômico mais baixo. Resultado inverso foi observado no padrão 4 (embutidos, ovos e carnes vermelhas); isto é, quanto maior o nivel socioeconômico menor a chance da adoção desse padrão. Tendência similar foi notada para o padrão 3 (frituras, doces, salgadinhos, refrigerante/ suco artificial).

Conclusões: padrões alimentares de crianças são dependentes das condições socioeconômicas das famílias e a adoção de itens alimentares mais saudáveis associa-se aos grupos de mais altos niveis socioeconômicos.

Palavras-chave Padrões alimentares, Condições socioeconômicas, Criança, Estudos transversais 


\section{Introdução}

O conhecimento dos padrões alimentares de crianças e a compreensão da sua relação com aspectos socioeconômicos podem proporcionar maior entendimento da complexa relação entre dieta e saúde na infância.

O padrão de consumo e os hábitos alimentares da população brasileira têm sofrido profundas mudanças ao longo do tempo, com maior tendência à adoção da dieta ocidental. Nota-se o consumo crescente de alimentos ricos em gorduras e carboidratos simples e a redução do consumo de carboidratos complexos, vitaminas e minerais. ${ }^{1}$

Diversos estudos têm demonstrado que variações nos padrões alimentares dos indivíduos podem ser influenciadas por fatores biológicos, nutricionais e socioeconômicos, tais como educação, renda e disponibilidade de alimentos; fatores comportamentais e estilo de vida.2,3 Para Sichieri et al., ${ }^{3}$ os fatores que mais explicaram o padrão de consumo alimentar, extensíveis às regiões Nordeste e Sudeste do país, foram escolaridade e renda.

Tradicionalmente, os estudos epidemiológicos que avaliam a dieta e sua relação com doenças o fazem tomando por base os efeitos de alimentos ou nutrientes específicos de maneira isolada. Contudo, sabe-se que os indivíduos não ingerem nutrientes isolados, mas refeições compostas de alimentos com complexas combinações de nutrientes que podem ter ações interativas e sinérgicas. ${ }^{4}$ A complexidade da dieta humana implica em uma abordagem mais ampla, concatenando o efeito das correlações entre alimentos e nutrientes. ${ }^{5}$ Assim, análises de identificação de padrões alimentares têm emergido como abordagem alternativa e complementar para avaliar a dieta, na qual padrões alimentares consistem em um “... conjunto ou grupos de alimentos consumidos por uma dada população"; 6 refletem exposição habitual de alimentos e nutrientes e são o meio preferido para acessar a relação entre dieta e doença. 5,7

Nas últimas décadas, a epidemiologia nutricional tem avançado com a utilização de diferentes técnicas estatísticas para avaliar o consumo alimentar de populações. Nessa perspectiva, sobressai-se a técnica da análise fatorial, com o método de extração dos componentes principais, a partir de um conjunto de dados do questionário de frequência alimentar (QFA), para determinar os padrões de consumo alimentar de uma população ou de grupos populacionais. 7,8

Assim, o presente estudo tem o objetivo de identificar os padrões de consumo alimentar de crianças de 4 a 11 anos, residentes na cidade do Salvador-
Bahia, com foco na exploração da relação entre fatores socioeconômicos e demográficos das crianças e de suas famílias e os padrões alimentares identificados.

\section{Métodos}

Trata-se de um estudo transversal, realizado na cidade de Salvador, capital da Bahia, Estado da região Nordeste do Brasil, com aproximadamente três milhões de habitantes. Foi realizado nos anos de 2005 e 2006 e é parte de uma ampla investigação voltada para o estudo dos fatores de risco para asma em Salvador, intitulada Social Changes, Asthma and Allergy in Latin America Programme (SCAALA), cujos aspectos metodológicos podem ser acessados em publicação anterior. ${ }^{9}$

As crianças incluídas neste estudo fazem parte de três coortes inicialmente formadas com o intuito de avaliar o impacto de um programa de saneamento ambiental sobre a ocorrência de diarréia na infância.9,10 A seleção dos participantes ocorreu de forma aleatória entre aqueles residentes em 24 áreas geográficas, selecionadas devido à complexidade e dimensão da cidade, onde os domicílios destas áreas foram aleatoriamente selecionados e eleita apenas uma criança por domicílio. $10 \mathrm{O}$ número amostral foi constituído por 1445 crianças e foi aplicado inquérito domiciliar desenvolvido pelo Instituto de Saúde Coletiva (ISC) da Universidade Federal da Bahia (UFBA).

Assim, o estudo aqui conduzido, inserido no âmbito do Programa SCAALA, contemplou inicialmente o total de 1445 crianças de 4 a 11 anos, permanecendo, para efeito de análises, 1260 crianças que dispunham de informações completas para as variáveis estudadas.

As informações socioeconômicas e demográficas foram fornecidas pelas mães ou responsáveis pelas crianças, às entrevistadoras treinadas. ${ }^{9}$ As variáveis socioeconômicas (escolaridade materna, posse de bens e utensílios domésticos) deram origem a um indicador socioeconômico, inicialmente idealizado por Alvarez et al. ${ }^{11} \mathrm{e}$, posteriormente, adaptado por Issler e Giugliani12 para a realidade brasileira. Assim, cada variável recebeu uma pontuação que variou de 0 a 4 , sendo o valor zero indicativo de pior situação socioeconômica. ${ }^{12}$ Após a somatória de todos os valores, o indicador obtido foi classificado em tercis: $1^{\circ}$ tercil representa nível socioeconômico mais baixo; $2^{\circ}$ tercil, intermediário e o $3^{\circ}$ tercil, mais alto. $\mathrm{O}$ indicador socioeconômico foi adotado como a variável de exposição principal, sendo o primeiro tercil a categoria de referência. As variáveis 
demográficas utilizadas foram: faixa etária materna ( $<30$ anos e $\geq 30$ anos), sexo (masculino, feminino) e faixa etária infantil ( $<8$ anos e $\geq 8$ anos).

Os dados de consumo alimentar foram registrados em um questionário de frequência alimentar (QFA) semi-quantitativo, aplicado às mães ou responsáveis pelas crianças, por nutricionistas e acadêmicos de nutrição, previamente treinados. As crianças maiores de oito anos de idade contribuíram com o fornecimento de seus próprios dados para o inquérito alimentar, informando sobre alimentos consumidos fora do ambiente domiciliar. Vale destacar que o QFA tem sido considerado o método de escolha para mensuração de consumo alimentar em estudos epidemiológicos de base populacional. 13 O referido questionário era composto por 98 alimentos, com escala de frequência alimentar abarcando cinco categorias, assim representadas: nunca consome, consome de uma a três vezes por mês, consome de uma a duas vezes por semana, consome de três a quatro vezes por semana, consome cinco ou mais vezes por semana. Os dados da frequência alimentar foram transformados em fração diária de consumo com o objetivo de se utilizar apenas uma unidade temporal. Posteriormente, os alimentos passaram a constituir 15 grupos com base nas características nutricionais e correlações obtidas através de análise fatorial preliminar. Tem-se os seguintes grupos constituídos: Grupo1- leite e derivados (leite integral, queijo amarelo, queijo branco, iogurte, leite fermentado, creme de leite, outros leites, leite desnatado); Grupo2-Catchup/ maionese/ mostarda (catchup, maionese, mostarda); Grupo3- embutidos (carne defumada, salsicha/ calabresa, presunto/ mortadela); Grupo4- frituras (churros, sonho, pastel e batata frita); Grupo5- carnes vermelhas (carne de boi, carne do sol, vísceras, carne de porco); Grupo6verduras e tubérculos (tomate, cenoura, abóbora, batata inglesa e batata doce); Grupo7- arroz/outros cereais (arroz, macarrão, bolo, biscoitos, pão francês, pão de leite, pão de milho, aveia, milho, farinha de milho, milho branco, milho em conserva, sucrilhos, farinha de cremogema, arroz integral, pão integral); Grupo8- leguminosas (amendoim cozido, amendoim torrado, soja texturizada e leite de soja); Grupo9- frutas (abacaxi, laranja, limão, lima, acerola, caju, umbu, maçã, morango, cajá, caqui, melancia, goiaba, manga, ameixa, mamão comum, mamão papaia, melão, abacate, banana, uva, sucos de fruta, coco, kiwi, ameixa fresca, nozes, castanha, cana-de-açúcar, caldo de cana); Grupo10- ovos (ovos); Grupo11- frango (frango); Grupo12pescados (mariscos, crustáceos, peixes, Leite de coco, azeite de dendê); Grupo13- salgadinhos (salgadinhos); Grupo14- refrigerantes/ sucos artificiais (refrigerantes e sucos industrializados); Grupo15- doces (gelatina, jujuba/bala/maria mole, picolé/geladinho, sorvete, pipoca doce, pipoca caramelizada, chocolates e achocolatados). Foram excluídos do agrupamento os alimentos que se mostraram outliers para a análise fatorial, 14 a exemplo da manteiga, margarina e do óleo de oliva.

Cada grupo de alimentos foi sintetizado em um único valor para cada criança, conformando uma medida resumo, expressa por meio da soma das frações diárias de cada alimento que constitui o grupo. Foi possível, dessa forma, obter uma variável contínua que foi padronizada, segundo escore $\mathrm{z}$ da normal padrão, e se constitui na variável de entrada para proceder a análise fatorial por componentes principais, para extração dos padrões alimentares. Os padrões alimentares consistem na variável desfecho, expressa em escores e categorizada em acima e abaixo da mediana (referência). O ponto de corte dado pela mediana para cada padrão alimentar foi de $-0,156$ (padrão 1 ), $-0,139$ (padrão 2), - 0,132 (padrão 3) e - 0,072 (padrão 4).

Para verificar a aplicabilidade e adequação do método multivariado de análise fatorial exploratória, com a técnica de análise de componentes principais, procedeu-se a estimação do teste de Kaiser-MeyerOlkin (KMO), cujo valor 0,8177 indicou a aplicabilidade do método. A adequação do tamanho da amostra também foi avaliada, tendo em vista a relação indivíduos/ itens alimentares, a qual foi de 13, portanto acima da faixa de 5 a 10 , mínimo recomendado.7,15 A rotação ortogonal Varimax 16 tem sido a mais empregada para determinação de padrões alimentares, 15,17 a qual também foi adotada no presente estudo, permitindo que os fatores resultantes não sejam correlacionados e melhorando a interpretabilidade dos dados.

O procedimento multivariado na análise fatorial permite que os itens alimentares contidos no QFA sejam agrupados segundo o grau de correlação existente entre eles, reduzindo a dimensionalidade dos dados e favorecendo a identificação de novas variáveis (fatores ou componentes). ${ }^{14}$ Para identificar o número de fatores retidos na análise fatorial, foram adotados os critérios de autovalores acima de um e o gráfico scree plot. Os alimentos ou grupos de alimentos que contribuíram para a caracterização de cada padrão apresentaram cargas fatoriais com valores iguais ou maiores a 0,30 , (considerando-se nível de significância de 0,05 e poder de $80 \%$ ) conforme recomendação de Hair et al.,18 sendo considerada a maior saturação da carga fatorial.

Para avaliar a associação do indicador socio- 
econômico e os padrões alimentares, foram construídos quatro modelos de regressão logística, uma vez que cada padrão alimentar identificado foi considerado uma variável dependente. Esses modelos foram ajustados por faixa etária e sexo da criança, faixa etária materna e ainda se levou em conta, no ajuste de cada modelo, os demais padrões alimentares, bem como a fração diária de consumo de manteiga e margarina. As análises foram realizadas, utilizando-se o software STATA (versão 9.0).

Este estudo foi aprovado pelo Comitê de Ética em Pesquisa do Instituto de Saúde Coletiva da Universidade Federal da Bahia, sob registro 00305/CEP-ISC. Após consentimento dos entrevistados, os dados foram coletados.

\section{Resultados}

Avaliando-se a caracterização da população de participantes do estudo, segundo variáveis demográficas, houve predomínio do sexo masculino $(53,4 \%)$, de crianças na faixa etária menor de oito anos $(55,1 \%)$ e de mães na faixa etária de 30 anos ou mais $(59,4 \%)$. Uma avaliação dos dados da característica da amostra em relação ao indicador socioeconômico revelou que crianças pertencentes às famílias com indicador socioeconômico alto apresentam predomínio de mães com 30 anos ou mais $(68,4 \%)$ em relação aos demais níveis socioeconômicos. Com relação à faixa etária e sexo, as crianças se distribuem de forma homogênea entre os três níveis do indicador socioeconômico.

Foram identificados, na análise fatorial por componentes principais, quatro fatores (padrões alimentares) com autovalores acima de 1 (um).

Os alimentos ou grupos de alimentos que contribuíram para a composição dos padrões de consumo alimentar, por apresentarem valores iguais ou maiores a 0,30 , considerada a maior saturação da carga fatorial, estão representados na Tabela 1.

Os quatro diferentes padrões de consumo alimentar que foram identificados para as crianças deste estudo explicaram $45,9 \%$ da variância total dos dados. O padrão 1 foi caracterizado pelo predomínio de frutas, verduras, leguminosas, cereais e pescados. O padrão 2 foi caracterizado pelo predomínio de leite e derivados, catchup/ maionese/ mostarda e frango. $\mathrm{O}$ padrão 3 foi caracterizado pelo predomínio de frituras, doces, salgadinhos, refrigerante/ suco artificial. O padrão 4 foi caracterizado pelo predomínio de embutidos, ovos e carnes vermelhas.

Tabela 1

Distribuição das cargas fatoriais para os quatro componentes (padrões alimentares) identificados na população estudada. Salvador, BA, 2005-2006.

\begin{tabular}{|c|c|c|c|c|}
\hline \multirow[t]{2}{*}{ Alimentos/ grupos de alimentos } & \multicolumn{4}{|c|}{ Padrões de consumo alimentar } \\
\hline & Padrão 1 & Padrão 2 & Padrão 3 & Padrão 4 \\
\hline Frutas & 0,74 & 0,22 & 0,09 & 0,12 \\
\hline Verduras & 0,58 & 0,07 & $-0,17$ & 0,33 \\
\hline Leite e derivados & 0,36 & 0,63 & 0,03 & $-0,06$ \\
\hline Catchup/maionese/mostarda & 0,02 & 0,70 & 0,12 & 0,04 \\
\hline Embutidos & 0,01 & 0,47 & 0,14 & 0,52 \\
\hline Frituras & 0,31 & 0,38 & 0,40 & $-0,08$ \\
\hline Frango & 0,11 & 0,34 & $-0,25$ & 0,08 \\
\hline Doces & 0,25 & 0,31 & 0,56 & 0,07 \\
\hline Leguminosas & 0,62 & $-0,20$ & 0,23 & $-0,02$ \\
\hline Cereais & 0,54 & 0,16 & 0,13 & 0,14 \\
\hline Ovos & 0,03 & 0,10 & 0,15 & 0,58 \\
\hline Carnes vermelhas & 0,21 & $-0,11$ & 0,06 & 0,72 \\
\hline Pescados & 0,56 & 0,21 & 0,00 & 0,05 \\
\hline Salgadinhos & 0,06 & $-0,07$ & 0,74 & 0,09 \\
\hline Refrigerante/suco artificial & $-0,06$ & 0,24 & 0,59 & 0,14 \\
\hline Autovalores & 3,20 & 1,39 & 1,14 & 1,04 \\
\hline \% explicação da variância & 14,90 & 11,45 & 10,70 & 8,90 \\
\hline \% variância acumulada & 14,90 & 26,30 & 37,01 & 45,90 \\
\hline
\end{tabular}

Método de extração: análise de componentes principais com rotação Varimax. Kaiser-Meyer-Olkin $(K M O)=0,8177$. 
A associação entre indicador socioeconômico e os padrões alimentares, ajustada por sexo, faixa etária da criança, faixa etária da mãe, escores dos padrões alimentares, manteiga e margarina, estão apresentados na Tabela 2.

A análise multivariada mostrou associação estatisticamente significante entre indicador socioeconômico e o padrão alimentar das crianças. As crianças pertencentes às famílias com indicador socioeconômico médio e alto apresentam maior chance do consumo alimentar ser caracterizado pelo padrão 1 (frutas, leguminosas, verduras, pescados e cereais) e pelo padrão 2 (leite/ derivados, catchup/ maionese/ mostarda e frango), quando comparadas às crianças de famílias com baixo indicador socioeconômico, mesmo após ajuste. Com relação ao padrão 3 , identificou-se que quanto maior o indicador socioeconômico há menor tendência das crianças consumirem os alimentos que compõem este padrão (frituras, doces, salgadinhos e refrigerante/suco artificial), embora a associação não tenha sido significante. Já para o padrão 4 , notou-se menor chance de consumo dos alimentos que o compõem (embutidos, ovos e carnes vermelhas) dentre as crianças de nível socioeconômico alto, comparadas às de nível socioeconômico baixo.

Tabela 2

Associação entre indicador socioeconômico e padrões de consumo alimentar da população estudada. Salvador, BA, 2005-2006.

\begin{tabular}{|c|c|c|c|c|c|c|c|c|c|}
\hline \multirow{2}{*}{$\begin{array}{l}\text { Indicador socio- } \\
\text { econômico }\end{array}$} & \multicolumn{9}{|c|}{ Padrão 1} \\
\hline & $N=1260$ & $\mathrm{n}$ & $\%$ & ORb & $p$ & IC95\% & OR 1 & $p$ & IC95\% \\
\hline Baixo & 474 & 178 & 37,6 & 1,00 & & & 1,00 & & \\
\hline Médio & 377 & 163 & 43,2 & 1,22 & 0,157 & $0,93-1,59$ & 1,17 & 0,286 & $0,88-1,55$ \\
\hline Alto & 409 & 211 & 51,6 & 1,71 & $<0,001$ & $1,32-2,23$ & 1,60 & 0,001 & $1,20-2,12$ \\
\hline \multirow{2}{*}{$\begin{array}{l}\text { Indicador socio- } \\
\text { econômico }\end{array}$} & \multicolumn{9}{|c|}{ Padrão 2} \\
\hline & $N=1260$ & $\mathrm{n}$ & $\%$ & ORb & $p$ & IC95\% & OR2 & $p$ & IC95\% \\
\hline Baixo & 474 & 140 & 29,5 & 1,00 & & & 1,00 & & \\
\hline Médio & 377 & 179 & 47,5 & 2,08 & $<0,001$ & $1,58-2,74$ & 2,14 & $<0,001$ & $1,60-2,86$ \\
\hline Alto & 409 & 232 & 56,7 & 3,74 & $<0,001$ & $2,31-3,99$ & 3,09 & $<0,001$ & $2,31-4,13$ \\
\hline \multirow{2}{*}{$\begin{array}{l}\text { Indicador socio- } \\
\text { econômico }\end{array}$} & \multicolumn{9}{|c|}{ Padrão 3} \\
\hline & $\mathrm{N}=1260$ & $\mathrm{n}$ & $\%$ & $\mathrm{OR}^{\mathrm{b}}$ & $p$ & IC95\% & $\mathrm{OR}^{3}$ & $p$ & IC95\% \\
\hline Baixo & 474 & 229 & 48,3 & 1,00 & & & 1,00 & & \\
\hline Médio & 377 & 168 & 44,6 & 0,88 & 0,336 & $0,67-1,15$ & 0,86 & 0,287 & $0,65-1,14$ \\
\hline Alto & 409 & 173 & 43,3 & 0,80 & 0,093 & $0,62-1,04$ & 0,78 & 0,094 & $0,59-1,04$ \\
\hline \multirow{2}{*}{$\begin{array}{l}\text { Indicador socio- } \\
\text { econômico }\end{array}$} & \multicolumn{9}{|c|}{ Padrão 4} \\
\hline & $\mathrm{N}=1260$ & $\mathrm{n}$ & $\%$ & $\mathrm{OR}^{\mathrm{b}}$ & $p$ & IC95\% & $\mathrm{OR}^{4}$ & $p$ & IC95\% \\
\hline Baixo & 474 & 237 & 50,0 & 1,00 & & & 1,00 & & \\
\hline Médio & 377 & 182 & 48,3 & 0,93 & 0,577 & $0,71-1,21$ & 0,90 & 0,436 & $0,64-1,18$ \\
\hline Alto & 409 & 176 & 43,0 & 0,74 & 0,027 & $0,57-0,97$ & 0,70 & 0,014 & $0,53-0,93$ \\
\hline
\end{tabular}

$\mathrm{OR}^{\mathrm{b}}=$ odds ratio bruto;

$\mathrm{OR}^{1}=$ ajustado por: sexo, faixa etária da criança, faixa etária da mãe, padrão 2, padrão 3 , padrão 4 , manteiga, margarina;

$\mathrm{OR}^{2}=$ ajustado por: sexo, faixa etária da criança, faixa etária da mãe, padrão 1, padrão 3, padrão 4, manteiga, margarina;

$\mathrm{OR}^{3}=$ ajustado por: sexo, faixa etária da criança, faixa etária da mãe, padrão 1, padrão 2 , padrão 4 , manteiga, margarina;

OR4= ajustado por: sexo, faixa etária da criança, faixa etária da mãe, padrão 1, padrão 2, padrão 3, manteiga, margarina. 


\section{Discussão}

Os dados deste estudo revelam que a caracterização dos padrões alimentares das crianças diverge segundo as condições socioeconômicas. Interessante notar que as crianças de famílias com nível socioeconômico médio e alto tiveram maior chance do consumo ser caracterizado pelos padrões alimentares 1 e 2 quando comparadas às crianças de famílias com baixo indicador socioeconômico. Ainda para as crianças de famílias com indicador socioeconômico médio e alto, observou-se menor chance do consumo alimentar ser caracterizado pelo padrão 4 e tendência, nessa mesma direção, para o consumo dos alimentos que integram o padrão 3, quando comparadas às crianças de famílias com baixa condição socioeconômica

Assim, dispor de melhores condições socioeconômicas implicaria em maior consumo de alimentos variados e, em sua maioria, saudáveis, a exemplo de frutas, verduras, leguminosas, cereais, pescados, leite e derivados, aves, dentre outros (padrões 1 e 2). Dispor de melhores condições socioeconômicas implica ainda em menor consumo de alimentos como embutidos, ovos e carnes vermelhas (padrão 4), além de uma menor tendência ao consumo de frituras, doces, salgadinhos e refrigerantes/ sucos artificiais (padrão 3).

Essa diferença na caracterização dos padrões alimentares, influenciada pelas condições socioeconômicas, remete à reflexão de quatro aspectos relevantes a serem abordados: primeiro, a questão do acesso ao alimento; segundo, as escolhas alimentares; terceiro, as mudanças nos padrões de consumo alimentar; quarto, as implicações na saúde e nutrição do grupo em questão.

A dificuldade de acesso aos alimentos por parte dos mais pobres, especialmente em países em desenvolvimento, revela que o aumento na insegurança alimentar não é reflexo da falta de alimentos. A Food Agriculture Organization (FAO), 19 em seu $10^{\circ}$ informe sobre a fome no mundo, sinaliza que os menos favorecidos são obrigados a restringir o consumo de alimentos, a variedade da dieta e a selecionarem alimentos mais baratos, nem sempre nutritivos. Nossos resultados estão de acordo com os achados desse estudo, uma vez que os padrões alimentares 1 e 2 abarcam alimentos como frutas, verduras, panificados, aves, leite e derivados com destaque de maior consumo para as crianças de famílias com melhores condições socioeconômicas. Os dados da Pesquisa de Orçamento Familiar $(\mathrm{POF})^{20}$ indicam que a aquisição alimentar per capita anual, na zona urbana, dá-se mais fortemente por aquisição monetária ao invés de aquisição não monetária, como ocorre na zona rural. Essa característica remete à questão da segurança alimentar nas grandes cidades, implicando em disponibilidade de condições socioeconômicas para o aceso ao alimento. Segundo resultados da $\mathrm{POF}, 20$ a aquisição alimentar domiciliar per capita anual por classe de rendimento monetário indicou que quanto maior a renda maior o consumo de frutas, bebidas não alcoólicas, leite e creme de leite, panificados, aves e menor o consumo de carnes, cereais e leguminosas. Nesse sentido, os dados da Pesquisa de Orçamento Familiar 2002-2003 indicam que a disponibilidade domiciliar de grande parte dos alimentos que constituem os padrões alimentares 1 e 2 , identificados em nossos achados, aumenta com a renda familiar. ${ }^{20} \mathrm{Um}$ outro estudo sobre insegurança alimentar intrafamiliar e perfil de consumo de alimentos, 21 concluiu que famílias em insegurança alimentar moderada ou grave apresentaram dieta monótona, basicamente composta por alimentos energéticos.

Os resultados estão ainda de acordo com estudo realizado por Perrin et al.,22 segundo o qual o padrão intitulado "prudente" foi caracterizado pelo consumo elevado de frutas, legumes, azeite, peixe e baixa ingestão de carnes. Neumann et al. 7 notaram que o padrão alimentar com baixos teores de açúcar simples e gorduras e elevado consumo de peixes predominou entre pessoas com maior renda e escolaridade. Lenz et al.23 também identificaram que padrões alimentares saudáveis foram mais frequentes entre mulheres com renda e nível educacional elevados. Vários estudos têm encontrado diferenças nos padrões de consumo alimentar segundo nível socioeconômico.2,22 Olinto et al. ${ }^{8}$ identificaram que indivíduos com baixa escolaridade materna e posição social ou que foram sempre pobres durante toda a vida tiveram uma elevada adesão ao padrão alimentar brasileiro comum; em contraste, o padrão de alimentos processados era mais provável de ser consumido por aqueles que pertenciam à média e alta posição social e que nunca foram pobres.

Os padrões de consumo alimentar são moldados pelas relações sociais, sendo influenciados pelas escolhas alimentares e pelo contexto social. $24 \mathrm{~A}$ natureza social da alimentação expressa tanto escolhas individuais com base na influência familiar, 25 quanto escolhas alimentares condicionadas pelas relações e pelo contexto social. $26 \mathrm{~A}$ estrutura social cria condições de práticas que podem constranger ou facilitar as escolhas, o que se aplica também às práticas alimentares. $\mathrm{O}$ escopo das 
possíveis práticas alimentares familiares inclui, desde a obtenção do alimento até o preparo e consumo. Nesse sentido, os resultados deste estudo concordam com os autores 24 na medida em que a obtenção do alimento foi constrangida ou facilitada pelas condições socioeconômicas, influenciando a caracterização dos padrões de consumo alimentar. O padrão 3, por exemplo, cujos alimentos têm maior saciedade e relativo baixo custo, tendeu a ser mais característico de crianças com baixo indicador socioeconômico. Por outro lado, crianças que apresentaram indicador socioeconômico médio e alto tiveram mais facilidade para obtenção de alimentos com custo mais elevado, a exemplo de leite e derivados, pescados, frutas e verduras, presentes nos padrões alimentares 1 e 2 . Compreende-se que, nos grupos sociais pertencentes aos níveis econômicos mais baixos, geralmente o orçamento familiar é mais restritivo e o consumo de frutas e verduras pode estar sub-valorizado ou sub-dimensionado em favor de alimentos com maior densidade energética e maior potencial de saciedade, a exemplo do que indicam os nossos resultados. Sabe-se que a família é relevante na influência de preferências alimentares das crianças, mas importa destacar que essa mesma família sofre interferência do contexto social em que vive.

Os padrões de consumo alimentar da população brasileira têm sofrido profundas mudanças ao longo do tempo, com ênfase especial a partir da década de noventa, junto ao processo de globalização e adesão à dieta ocidental. 1 Muitos alimentos consumidos pela população aqui estudada estão em consonância com observações registradas por Vasconcelos. 1 Observa-se, por exemplo, que o consumo de alimentos industrializados constitui muitos dos padrões alimentares identificados entre as crianças investigadas, a exemplo de catchup, maionese e mostarda no padrão 2; salgadinhos, refrigerantes/ sucos artificiais e frituras no padrão 3; embutidos, como salsicha, calabresa, presunto e mortadela, no padrão 4. Assim, mesmo que o consumo alimentar dessas crianças tenha maior explicação da variabilidade dos dados conferida pelos padrões alimentares 1 e 2, de maneira geral, os padrões são permeados pela presença de alimentos industrializados, tidos como não saudáveis, confirmando, na população estudada, a tendência de mudança nos padrões alimentares já visualizada para a população brasileira.

Os dados deste estudo tendem a mostrar um cenário de consumo para crianças com baixas condições socioeconômicas, em que estão presentes alimentos industrializados, ricos em açúcares simples e gorduras (doces, salgadinhos, refrigerantes e frituras), além de reduzido consumo de verduras e frutas, antioxidantes dietéticos e ácidos graxos poliinsaturados, configurando-se um cenário propício ao desenvolvimento de doenças não transmissíveis. ${ }^{27}$ Ganham destaque na atualidade outros problemas de saúde, como asma e atopia, relacionados a um cenário alimentar como o acima descrito. 28

Assim, a identificação de padrões de consumo alimentar de crianças poderá fornecer subsídio para o redirecionamento das políticas públicas no âmbito da área de alimentação e nutrição no país, ${ }^{29}$ reorientando práticas alimentares saudáveis, sem perder de vista as condições socioeconômicas desse público alvo.

Cabe comentar ainda sobre as limitações deste estudo. Uma delas diz respeito ao viés de memória que impregna as informações sobre o consumo alimentar. Nesse sentido, a adoção de um álbum contendo as dimensões dos alimentos e conteúdo consumido em tamanho real, como utilizado nesta investigação, diminui os efeitos desse viés sobre os dados fornecidos. 30 Com o intuito de reduzir os possíveis efeitos do viés de informação, fomentou-se a participação das crianças, com faixa etária maior de oito anos no momento da aplicação do QFA, para contribuir com os dados sobre o consumo alimentar realizado fora da unidade domiciliar. Outra limitação diz respeito ao caráter da transversalidade dos dados que impede que inferências mais consistentes e abrangentes possam ser realizadas, neste sentido, destaca-se que nenhuma relação de causa e efeito está sendo destacada neste estudo. Ademais dessas limitações, há que se levar em consideração o uso da análise fatorial com a carga de subjetividade envolvida na definição do número de fatores a serem extraídos. ${ }^{14,17}$ Assim, independentemente das limitações consideradas, entende-se que o presente estudo se torna importante para compreender e explicar a relação entre condições socioeconômicas e padrões alimentares, bem como a identificação de grupos populacionais em risco por integrar ao seu padrão de consumo, alimentos que não contribuem para manter o adequado estado de saúde e nutrição.

Assim, pode-se concluir que os padrões alimentares de crianças em uma área urbana são fortemente dependentes das condições socioeconômicas das famílias. Essas condições constrangem ou facilitam as escolhas alimentares, e a adoção de padrões com itens alimentares mais saudáveis se associa aos mais altos níveis socioeconômicos da criança. 


\section{Agradecimentos}

Às famílias das crianças que participaram deste estudo. Ao Instituo de Saúde Coletiva, no âmbito do SCAALA- Social Changes, Asthma and Allergy in Latin America Programme, pelo apoio financeiro do The Wellcome Trust e do Conselho Nacional de Desenvolvimento Científico e Tecnológico - CNPQ (Programa de Pesquisa para o SUS-PPSUS), bem como pela infra-estrutura cedida para o desenvolvi-

\section{Referências}

1. Vasconcelos FAG. Tendências históricas dos estudos dietéticos no Brasil. História, Ciências, Saúde. 2007; 14: 97-219.

2. Irala-Estévez J, Groth M, Johansson L, Oltersdorf U, Prättälä R, Martínez-González MA. A systematic review of socio-economic differences in food habits in Europe: consumption of fruit and vegetables. Eur J Clin Nutr. 2000; 54: 706-714

3. Sichieri R, Castro JFG, Moura AS. Fatores associados ao padrão de consumo alimentar da população brasileira urbana. Cad Saúde Pública. 2003; 19: S47-53.

4. Jacobs DRJ, Steffen LM. Nutrients, food and dietary patterns as exposures in research: a framework for food synergy. Am J Clin Nutr. 2003; 78: S508-13.

5. Hu FB. Dietary pattern analysis: a new direction in nutritional epidemiology. Curr Opin Lipidol. 2002; 13: 3-9.

6. Garcia RWD. A comida, a dieta, o gosto: mudanças na cultura alimentar urbana [tese]. São Paulo: Instituto de Psicologia da Universidade de São Paulo; 1999.

7. Neumann AICP, Martins IS, Marcopito LF, Araujo EAC. Padrões alimentares associados a fatores de risco para doenças cardiovasculares entre residentes de um município brasileiro. Rev Panam Salud Publica. 2007; 22: 329-39.

8. Olinto MT, Willett WC, Gigante DP, Victora CG Sociodemographic and lifestyle characteristics in relation to dietary patterns among young Brazilian adults. Public Health Nutr. 2010; 25: 1-1

9. Barreto ML, Cunha SS, Alcântara-Neves N, Carvalho LP, Cruz AA, Stein RT, Genser B, Cooper PJ, Rodrigues LC. Risk factors and immunological pathways for asthma and other allergic diseases in children: background and methodology of a longitudinal study in a large urban center in Northeastern Brazil (Salvador-SCAALA study). BMC Pulm Med. 2006; 6: 1-15.

10. Teixeira MG, Barreto ML, Costa MCN, Strina A, Martins DJ, Prado M. Sentinel areas: a strategy for public health montoring. Cad Saúde Pública. 2002; 18: 1189-95.

11. Alvarez ML, Muzzo S, Ivanovic D. Escala para mediciòn del nìvel socioeconômico, en el área de la salud. Rev Med Chile. 1985; 113: 243-9.

12. Issler RMS, Giugliani ERJ. Identificação de grupos mais vulneráveis a desnutrição infantil pela medição do nível de pobreza. J Pediatr (Rio J). 1997; 73: 101-5. mento do trabalho. Aos colaboradores: S. D'Innocenzo como autor principal na elaboração do manuscrito; M.L. Barreto e L.C. Rodrigues como coordenadores no planejamento do estudo e levantamento de recursos financeiros; S. D'Innocenzo, M.S. Prado, S.M.A. Matos, A.M.O. Assis e L.R. Sampaio no planejamento da coleta de dados; S. D'Innocenzo, M.S. Prado, S.R.S. Pereira, A.P. Barros na realização da análise estatística. Todos os autores contribuíram para revisão do artigo final.
13. Willet W. C. Nutritional epidemiology. New York: Oxford University Press; 1998.

14. Mingoti SA. Análise de dados através de métodos de estatística multivariada: uma abordagem aplicada. Belo Horizonte: Ed. UFMG; 2007.

15. Olinto MTA. Padrões alimentares: análise de componentes principais. In: Kac G, org. Epidemiologia nutricional. Rio de Janeiro: Fiocruz/ Atheneu; 2007. p. 213-62.

16. Kaiser, HF. The varimax criterion for analytic rotation in factor analysis. Psychometrika. 1958; 23: 187-200.

17. Newby PK, Tucker KL. Empirically derived eating patterns using factor or cluster analisys: a review. Nutr Rev. 2004; 62: 177-203.

18. Hair JrJF, Anderson RE, Tatham RL, Black WC. Análise multivariada de dados. 9 ed. Porto Alegre: Bookman; 2005.

19. FAO (Organización de las Naciones Unidas para la Agricultura y la Alimentación). El estado de la inseguridad alimentaria en el mondo. Crisis económicas: repercusiones y enseñanzas extraídas. Subdivisión de Políticas y Apoyo en Materia de Publicación Electrónica. División de Comunicación. Roma: FAO; 2009. [Acesso em 12 jun 2010]. Disponível em: www.earthprint.com/product focus.php?id=FAO110523

20. IBGE (Instituto Brasileiro de Geografia e Estatística). Pesquisa de orçamentos familiares 2002-2003. [Acesso em 15 abr 2010]. Disponível em: www.ibge.gov.br/home/estatistica/populacao/condicaodevida/pof/2002analise/analise. pdf

21. Panigassi G, Segall-Correa AM, Marin-Leòn L, PèrezEscamilla R, Maranha LK, Sampaio MFA. Insegurança alimentar intrafamiliar e perfil de consumo de alimentos. Rev Nutr. 2008; 21 (Suppl 1): S135-44.

22. Perrin AE, Dallongeville J, Ducimetiere P, Ruidavets JB, Schlienger JL, Arveiler D, Simon C. Interactions between traditional regional determinants and socio-economic status on dietary patterns in a sample of French men. Br J Nutr. 2005; 93: 109-14.

23. Lenz A, Olinto MTA, Dias-da-Costa JS, Alves AL, Balbinotti M, Pattussi MP, Bassani DG. Socioeconomic, demographic and lifestyle factors associated with dietary patterns of women living in Southern Brazil. Cad Saúde Pública. 2009; 25: 1297-1306. 
24. Delormier T, Frohlich KL, Potvin L. Food and eating as social practice: understanding eating patterns as social phenomena and implications for public health. Sociol Health Illn. 2009; 31: 215-28.

25. Bourdieu P.Esboço de uma teoria da prática. In: Ortiz R, editor. Pierre Bourdieu: Sociologia. São Paulo: Ática; 1983. p. $46-81$.

26. Giddens A. The constitution of society. Berkeley and Los Angeles: University of California Press; 1984

27. Santos CRB, Portella ES, Avila SS, Soares EA. Fatores dietéticos na prevenção e tratamento de comorbidades associadas à síndrome metabólica. Rev Nutr. 2006; 19: 389-401 .
28. Devereux G, Seaton A. Diet as risk factor for atopy and asthma. J Allergy Clin Immunol. 2005; 115: 1109-17.

29. Brasil. Ministério da Saúde. Secretaria de Atenção à Saúde. Departamento de Atenção Básica. Política nacional de alimentação e nutrição. (Série B. Textos Básicos de Saúde). Brasília, DF; 2003.

30. Magalhães LP, Oliveira VA, Santos NS, Araújo CS. Guia prático para estimativa de consumo alimentar. Salvador: UFBA; 2000

Recebido em 22 de outubro de 2010

Versão final apresentada em 30 de dezembro de 2010

Aprovado em 31 de janeiro de 2011 